\title{
THE VELOCITY OF IONS FROM HOT PLATINUM WIRES. II.
}

By C. D. Child.

$\mathrm{HE}$ second part of this article deals principally with the dis-
charge from a hot platinum wire in other gases than air and in a vacuum. The work is not at all a complete investigation, but I shall not be able to continue it further at present, and therefore publish the experiments which have already been performed.

But first I wish to describe a few experiments with the particles driven from the wire. The presence about the wire of particles which diminish the velocity of ions has already been shown. It was noticed that the copper wires to which the platinum was attached became oxidized and that there was thus a possibility that particles of copper oxide were producing the effect. The copper wire was therefore removed and only platinum wire was used in the enclosed tube. The decrease in the amount of discharge was then even more marked than before.

A second test regarding the same point was then made by heating the copper wire and then examining the air about it for the presence of nuclei in the formation of clouds. Such nuclei were indeed found when the copper was sufficiently heated, but the amount of current which had been used in former experiments was not sufficient to heat the copper which was used, so as to give off such particles. This seems to be sufficient to show that those which produce the decrease in the rate of discharge came from the platinum itself.

The effect of these particles on the ions coming from a flame was examined. Several turns of platinum wire were placed between pieces of wood about $1.5 \mathrm{~cm}$. apart, and $10 \mathrm{~cm}$. long. The air arising from these wires was passed between two pieces of tin of the same length and the same distance apart and $6 \mathrm{~cm}$. in width. These were kept at a potential difference of a hundred volts. They 
C. D. CHILD. [Vol. XIV.
[Vol

were for the purpose of taking any ions out of the air arising from the platinum. The air was then allowed to pass between a flame kept at 50 volts and a circular plate $5 \mathrm{~cm}$. in diameter surrounded by a guard ring. The plate was about $2 \mathrm{~cm}$. from the flame.

A charged plate was first put in the place of the flame and no discharge was noted when the wire beneath was heated. This showed that the pieces of tin were properly serving their purpose of drawing the ions out of the ascending air.

The effect of the ascending air on the discharge from the flame was then noted. When the flame was charged positively and the wire beneath was not heated, the rate of discharge was $8 \times \mathrm{IO}^{-10}$ ampère. When the wire was heated it was $4 \times 10^{-10}$ ampère, showing a decrease of 50 per cent. caused by the particles arising from the platinum. When the flame was discharged negatively, the rate of discharge was $12 \times 10^{-10}$ ampère, and with the wire heated $3.2 \times$ $10^{-10}$ ampère, a decrease of 83 per cent. This experiment was modified in various ways, but the decrease in the velocity of the negative ions was always greater than that of the positive.

It seems most reasonable that this should be so. Discharge occurs from positive platinum wires at a lower temperature than from a negative one. Apparently there is greater attraction between the platinum and negative ions than between platinum and positive ions. If this be true, we must expect a greater attraction between the particles of platinum given off and the negative ions. This experiment showing the effect of the particles on the ions from a flame bears out this idea.

And it also seems reasonable that this should be the explanation for the fact that in case of discharge from hot wires the negative ions move more slowly than the positive. In most cases of discharges through gas the negative ions move more rapidly. Some explanation of the behavior here is desirable. If the particles which are driven off have a greater affinity for the negative ions, they will naturally load them to a greater extent than the positive and cause them to move more slowly. As will be shown shortly, we have reason to suppose that the particles of platinum are not driven off to so great an extent in hydrogen as in air and there we find in general that the negative ions move the more rapidly. This fact 
also indicates that the presence of the particles is the cause of the slow movement of the negative ions.

The phenomena could also be explained by assuming that the ions are formed from different substances and that the positive ions are given off from some substance which produces more rapidly moving ions. Indeed for some time I supposed that some such explanation was the correct one; but in view of all the facts which have been observed it does not seem as reasonable an explanation as the view given above.

This may throw some light on the fact that the positive ions drawn from the arc move more rapidly than the negative ones, but I hope to publish soon some experiments on the discharge from carbon and the discussion of that point may properly be left until then.

Discharge in Hydrogen.-It has been found that platinum does not decrease in weight when heated in hydrogen. ${ }^{1}$ The discharge from a wire surrounded by hydrogen was therefore examined, but it gave decided evidence of the presence of particles which loaded the ions and diminished their velocity. The rates of discharge with different currents through the wire are given in Table XV. The potential difference was 30 volts. The same cylinder and the same glass tube were used in these experiments that have been described in connection with Fig. 5, Part I.

TABLE XV.

\begin{tabular}{|c|c|c|c|}
\hline Current through Wire. & + Discharge. & - Discharge. & \\
\hline 6.5 & $.07 \times 10^{-8}$ & & \\
\hline 7 & $.35 \quad 6$ & & \\
\hline 7.5 & $.63 ،$ & $1.2 \times 10^{-1} 8$ & \\
\hline 8 & 1.056 & 7.16 & \\
\hline 8.5 & $1.5 \quad 6$ & 8.86 & \\
\hline 9 & 1.5 & 86 & \\
\hline 9.5 & 1.5 & 3.4 & \\
\hline 10 & 1.5 ، & 1.56 & $\bullet$ \\
\hline
\end{tabular}

In attempting to record a higher temperature the wire was melted. The positive discharge occurs at lower temperatures than the negative as in air. This may possibly be due to a film of oxygen

1 Wied. Ann., 35, 107. 
about the platinum which hinders the negative discharge from passing from the wire, but this does not seem a probable explanation since the wire was heated to nearly white heat in the hydrogen for thirty minutes without producing any change in this respect. However in view of the difficulty which Spiers ${ }^{1}$ has shown to exist in getting rid of the oxygen film one can not be positive that thus heating the wire would have the desired effect.

The negative discharge is in general larger than the positive. There was, however, a very great decrease in the amount of discharge when the current through the wire was increased from nine ampères to ten. In fact the negative discharge became as small as the positive. This seemed surprising, and another wire was substituted for the one which had been melted. This second piece required a little more current to bring it to the desired temperature, but it showed exactly the same peculiarities as the first.

The wire was heated for several minutes at as high a temperature as possible, but the discharge with the smaller current continued to be as large as ever and to decrease again when the temperature was raised.

In air the rate of discharge when the wire was first heated was larger than it was after the current had been passing for some time. I endeavored to show that the same was true in hydrogen, but the needle of the galvanometer which I was using for these observations required about fifteen seconds to come to rest, and by that time the discharge had reached a constant value.

Though other experimenters have found no decrease in the weight of platinum when heated in hydrogen, it seemed well to test the matter again, using a current as large as that which produced the smaller rate of discharge. But when the wire was heated for about twenty minutes this piece was also melted. The two parts were, however, weighed and their weight was found to be the same to within .I mg. as that of the wire before it had been heated. It did not seem profitable to repeat further the work which has already been performed on this point.

But this does not, of course, prove that no particles are driven off, since we know that they are driven off when the wire is in air at

1 Phil. Mag. (5), 49, 84. 
temperatures too low to cause any appreciable loss in weight. The hydrogen about the wire was, therefore, examined for nuclei with water condensation. Moist hydrogen was passed into the tube and then suddenly drawn off and the cloud formation was as noticeable as in air, even though the wire was not heated above red heat. After the condensation had been produced a few times it ceased to be visible until the wire was again heated. Evidently the formation of cloud is a more delicate test of the presence of particles than either the loss in weight or the decrease in the velocity of the ions.

This experiment seems to make it probable that the decrease in the velocity of the ions was caused by particles driven off from the platinum and loading the ions. The particles probably are not driven off to as great an extent as they are in air, since no loss of weight has been detected, and since the velocity of the ions in the hydrogen is much greater than it is in the air.

The fact that the negative discharge in the hydrogen was greatly affected while the positive was not indicates the same thing. If a new kind of ionization was being produced at the higher temperatures, we should expect some indication of its presence in the positive discharge. On the other hand we have already had evidence to show that the particles driven from the platinum have a greater attraction for the negative ions than for the positive, and this would easily explain the phenomena as they here occur.

The particles sent off in hydrogen are no doubt platinum and not an oxide. It would seem highly improbable that a platinum oxide would be formed in the presence of hydrogen. It seems, therefore, probable that the particles sent off in the air are also particles of platinum instead of any oxide of platinum.

Observations were taken with different potential differences and the rate of discharge was found to vary much as it did in air.

This fact seems to show us that the velocities may here be computed as they were in air. When this is done the highest value of $K$, the velocity of the positive ions, as computed from Table XVIII. was found to be $32.6 \mathrm{~cm}$. per second as compared with $4.5 \mathrm{~cm}$. in air, and that of the negative ions $195 \mathrm{~cm}$. as compared with 3.5 . These values seem to be very large, but the data taken certainly indicate such velocities. 
Discharge in Oxygen, $\mathrm{CO}_{2}$ and $\mathrm{CO}$.-With other gases than hydrogen the decrease in the rate of discharge from its first value was very much the same that it was in air. The discharge in oxygen became even smaller than in air enclosed in the same tube. The velocity of the ions was apparently only $.2 \mathrm{~cm}$. per second, but no importance can be given to this value, since it depended to so great an extent on the size of the tube and length of time that the wire was heated.

The discharge in $\mathrm{CO}_{2}$ behaved very much the same as it did in air. In $\mathrm{CO}$ the discharge did not decrease as much as in air, but still the behavior of the discharge in this gas was much more similar to its behavior in air than to that in hydrogen. No attempt was made to secure any of these gases chemically pure except hydrogen. In that case no difference was seen between the discharge in hydrogen made from commercial $\mathrm{HCl}$ and zinc, and that made from chemically pure $\mathrm{HCl}$ and zinc.

Discharge in a Vacuum. - I then proceeded to examine the rate of discharge in a vacuum. It has been shown by others that the rate of discharge from a hot platinum wire increases as the pressure of the surrounding gas is diminished. This was first verified, but in doing so it was found that the discharge at any given pressure of the gas was greatest when the wire was heated for the first time at that particular temperature. In one of the first experiments with the pressure of about $\mathrm{I} \mathrm{mm}$., with a current of 5 ampères passing through the wire, and a potential difference between the wire and the cylinder of 40 volts the discharge at the beginning was fully $8.4 \times 10^{-7}$ ampère. This fell in a few minutes to $4.2 \times 10^{-8}$ ampère, only $\frac{1}{20}$ of its first value. These measurements were taken with a galvanometer whose constant was $4.2 \times 10^{-8}$ ampère per scale division.

At first sight this would seem to be nothing different from what was found at atmospheric pressure. We there found the rate of discharge to diminish as the wire was heated in a closed tube for some time. The same thing occurs here but to a more marked degree. But further examination seemed to indicate that this phenomenon was essentially different from the other. Allowing the air to enter the tube and then drawing it off did not at all bring the 
wire back to its original condition. It no longer gave off as much discharge as it did in the beginning. Allowing the wire to remain over night undisturbed did not restore its power of discharge.

Effect of Hydrogen on the Wire.-Moreover heating the wire in a flame from gasolene restored it partially to its original condition and heating it to a white heat in hydrogen almost entirely restored it. But before any exact measurements were taken with this wire it was accidentally melted and another one was put in its place. The second wire behaved quite differently from the first. The rate of discharge was but little larger when it was first heated in a vacuum from what it was afterwards. Heating it in hydrogen had but little effect upon it. Both moist and dry hydrogen were tried, but neither of them affected the wire. It seemed as if some mistake must have been made in the first observations, but upon trying different pieces of platinum it was found that there was much variety in their behavior.

Platinum was accordingly secured from three different sources. It all contained more or less iridium. Two pieces which were said to contain approximately $\mathrm{I} / 2$ per cent. and 5 per cent. of iridium showed a decrease from about $7 \times 10^{-7}$ ampère to $1.7 \times 10^{-7}$ ampère when first heated, the potential difference being 50 volts and the current through the wire 5 ampères. The third, containing approximately ro per cent. of iridium gave a discharge at first of over $8.4 \times 10^{-6}$ ampère, and then decreased to about the same as the others. All three of these were brought back to nearly their original condition by being heated in hydrogen and they were not affected by being heated in air.

All of the wires were heated in the air at atmospheric pressure for a few minutes before they were heated in a vacuum, so that we would expect any dirt to have been burnt off. But even if one were to assume that the effect was due to some impurity in the wire this alone would not explain the fact that the hydrogen had so great an effect in restoring the wire to its original condition.

A piece of wire having 5 per cent. of impurity, the kind of impurity not being given, was then tried and this was found to have a greater rate of discharge at the beginning than any of the others and a greater subsequent decrease. Heating it in hydrogen produced a 
large increase in the rate of discharge, although it lacked much of bringing it back to its original condition.

A piece of wire having to per cent. of impurity showed a smaller rate of discharge at first and a smaller change from this than the wire containing only 5 per cent. of impurity.

No attempt was made to go further with this part of the work. The difference in behavior in the different pieces of wire may have been due to a very slight impurity or possibly to gases previously absorbed by the wire. It would probably be a long and tedious piece of investigation to trace out the cause of this behavior and I am unable at present to perform it.

Experiments with Palladium.-Palladium is known to absorb greater quantities of hydrogen than platinum. A few experiments were, therefore, performed with it. But palladium melts at a lower temperature than platinum and on that account no very satisfactory results could be obtained. It melted before any negative discharge was produced and it seemed to be especially liable to melt when heated in hydrogen. As far as was found palladium behaved the same as platinum. The discharge was largest at first, decreased after a short time and was largely restored by heating in hydrogen. The final rate of discharge from it was about the same as that from platinum.

The effect produced by heating the wire in hydrogen throws some light on the mechanism of discharge, but the discussion of this will be deferred until other experiments have been described.

Observations were then made on the rate of discharge in a vacuum under varying conditions. No great exactness can be expected here since the rate of discharge depends to such a large extent upon the length of time that the wire has been heated in a vacuum, but after a few minutes the rate of discharge became reasonably constant and observations could be made of the effects produced by varying the other conditions.

The Current Varied with Wire in Vacuum.-The first quantity varied was the amount of current flowing through the wire. The potential difference was 45 volts and the pressure of the air I $\mathrm{mm}$. The other conditions were the same as in the preceding experiments. The results of these observations are given in Table XVI. Column 
I gives the amount of current flowing through the wire, column 2 the positive discharge measured in ampères, column 3 the negative.

TABLE XVI.

\begin{tabular}{|c|c|c|}
\hline Current through Wire. & + Discharge. & - Discharge. \\
\hline 4.3 & $.42 \times 10^{-8}$ & $.21 \times 10^{-8}$ \\
\hline 4.5 & $.63 ،$ & $1 \quad$ " \\
\hline 4.8 & 1.6 & 3.7 \\
\hline 5 & 3.3 & 10 \\
\hline 5.2 & 6.6 & 22 \\
\hline
\end{tabular}

With larger currents the wire was liable to melt. The same current, of course, produces a higher temperature in a vacuum than in air.

The negative current is larger under these conditions. It is also noticeable that the effect of increasing the current is not the same when the wire is in a vacuum that it is in air at ordinary pressures. In air the discharge soon reached a maximum, while here it continually increases.

Potential Varied.-A still greater difference between the phenomena in air and in a vacuum was shown when the potential difference between the wire and the cylinder was varied. Table XVII. gives the rates of discharge with different potential differences. The current was 5.2 ampères and the other conditions were the same as in the preceding experiment. Column I gives the potential difference between the wire and the cylinder, column 2 the rate of positive discharge measured in ampères, column 3 that of the negative.

TABLe XVII.

\begin{tabular}{|c|c|c|}
\hline Potential Difference. & + Uischarge. & -Discharge. \\
\hline 45 & $4.7 \times 10^{-8}$ & $19 \times 10^{-8}$ \\
\hline 36 & $3.6 \quad$ ، & 15 ، \\
\hline 26 & $3.6 \quad 6$ & $10 " ،$ \\
\hline 18 & 3.1 & 8.4 “ \\
\hline 9 & 2.9 & $8.4 " 6$ \\
\hline 5 & 2.5 & $8.4 " 6$ \\
\hline 1.5 & 1.9 " & $8.2 "$ \\
\hline
\end{tabular}

In the last reading the ground was connected to one end of the wire running into the tube. A shunt could have been placed about 
the wire and different points on this grounded, but this would have made one part of the wire positive and the rest negative. No conclusion could have been drawn from observations thus made.

. The current here decreased but very little while the potential difference was changed from 45 to 1.5 volts. This was entirely different from the decrease shown when the potential difference was diminished at atmospheric pressure. The discharge at that time decreased very rapidly.

An attempt was made to determine the potential gradient between the hot wire and the surrounding cylinder by means of a second wire. The difficulty to in finding the potential by this means has already been pointed out ${ }^{1}$ and in this case the difficulty appeared to be insuperable. All of the space within the tube becomes filled with ions. Moreover the surface of the insulator within the tube ceases to some extent to insulate when the discharge is taking place.

The observations taken seemed to indicate that the potential about the wire approaches the potential of the wire when the pressure of the gas is diminished, but after some study of the leakage to the exploring wire, I decided that it was impossible to make any definite statement concerning it.

Such a change in the potential as that which appeared to take place, could be explained by supposing that all of the space within the tube becomes partially ionized. Even at atmospheric pressures the air becomes ionized to more than molecular distances and the same thing may occur to a much greater extent in a vacuum. But it would be useless to attempt to decide this point, until we have more definite knowledge of the condition of the gas.

The Velocity of Ions in a Vacuum.-The way in which the discharge varies when the potential difference is varied is of itself sufficient to show us that the mechanism of discharge is not the same in a vacuum that it is in air at ordinary pressures. Either the velocity of the ions is so great that their presence does not greatly affect the potential about the wire, or the ionization occurs throughout the gas. In either case the task of finding the velocity of the ions in a vacuum is very difficult. The velocity can not be determined by blowing air past the wire. And until we know whether

1 Phys. Rev., 12, 67. 
the ionization all takes place at the surface of the wire or throughout the gas the employment of an alternating potential difference would give us no definite knowledge.

I had intended to use the method depending upon the determination of the rate of discharge and of the potential gradient about the wire. But evidently we have no right to assume that the potential gradient is the same here that it is in air at higher pressures. Even when the potential difference was only I. 5 volts the rate of discharge did not vary at all as it did at atmospheric pressure.

The water dropper can not be used to determine the potential gradient and the attempt to determine this by a wire has been described.

The method which has been used by J. J. Thomson ${ }^{1}$ in finding the velocity of the ions could perhaps be used, but it would require much care. Among other measurements the kinetic energy of the ions carrying the discharge must be determined. As far as can be foreseen this would be much smaller in the case of discharge from a wire than in the case of cathode discharge. The work might possibly be performed, but I have made no attempt whatever to do so.

The Vacuum Varied.-When the attempt was made to secure a series of readings with different pressures of the gas, it was found that no two sets were ever identical. In the first place the work was complicated by the cooling effect of the convection currents about the wire. The same current through it raises it to a higher temperature when there is less gas present. In making observations the temperature should be kept constant. In order to do this a high resistance galvanometer was place in shunt with the wire, and the potential difference at the ends of the wire was measured by the galvanometer. It was assumed that the temperature would be constant, if the resistance were constant, and the resistance is proportional to the ratio of the potential difference measured by the galvanometer and the current through the wire measured by an ammeter. In making the observations this ratio was, accordingly, kept constant.

But even with this precaution I secured widely different sets of readings. At atmospheric pressure the discharge depends largely upon the number of particles about the wire, and with very low

${ }^{1}$ Phil. Mag. (5), 44, 302. 
pressures it appears to depend on the amount of occluded hydrogen. These probably were the causes of the wide divergence in the data secured. However, the data which were taken in one case are given in Table XVIII. At each pressure of the gas the wire was heated until the discharge appeared to be constant.

It was found that with lower pressures the particles did not appear to check the motion of the ions. At least the discharge did not then become smaller when the wire was heated for some time. Thus for the particular wire used for securing the data given in Table XVIII. the discharge decreased with time until the pressure was diminished from $50 \mathrm{~cm}$. to $40 \mathrm{~cm}$. After that it continued to be large without regard to the length of time that the wire was heated.

Different pieces of wire behaved differently in this respect as in other respects, some of them apparently sending out the particles at lower pressures than others. In fact it became apparent that it would be useless to attempt to secure exact data on any of these phenomena without a thorough study of the impurities that may exist in the wire.

Column I in Table XVIII. gives the pressure of the gas in $\mathrm{cm}$. of mercury. Column 2 the current through the wire, column 3 the positive discharge in ampères, column 4 the negative. The potential difference between the wire and the cylinder was 30 volts. The cylinder about the wire was the same that had been used in preceding experiments.

TABLE XVIII.

\begin{tabular}{|c|c|c|c|c|c|}
\hline \multirow{2}{*}{$\frac{\text { Pressure of Gas. }}{72}$} & \multirow{2}{*}{$\frac{\text { Current through Wire. }}{6.2}$} & \multicolumn{2}{|c|}{ + Discharge. } & \multicolumn{2}{|c|}{ - Discharge. } \\
\hline & & .056 & $10^{-9}$ & .03 & $10^{-9}$ \\
\hline 50 & 6.0 & .086 & “ & .05 & “ \\
\hline 40 & 5.9 & .58 & “ & .30 & “ \\
\hline 30 & 5.8 & 1.4 & “ & .84 & “ \\
\hline 20 & 5.7 & 3.1 & “ & 3.7 & “ \\
\hline 10 & 5.7 & 6.3 & " & 10. & " \\
\hline 5 & 5.6 & 14. & " & 25. & “ \\
\hline 2 & 5.5 & 28. & " & 50. & " \\
\hline 1 & 5.4 & 50. & “ & 160. & “ \\
\hline .5 & 5.3 & 160. & " & 360. & “ \\
\hline
\end{tabular}

These data agree with those of others in showing that the negative discharge is greater than the positive when the wire is in a vacuum and heated sufficiently high. 
A smaller tube was hermetically sealed and the gas exhausted until cathode rays were produced. The behavior of this tube was but little different from the preceding. The first discharge was very large and continued to decrease for a long time, the final value being little larger than the value it had at I $\mathrm{mm}$. pressure. The size of the cylinder also had little to do with the amount of discharge.

The Mechanism of Discharge.-It has been suggested that the discharge is caused by dirt on the wire. This may have some effect on the large discharge produced when the wire is first heated, but it hardly seems possible that it can be the only cause of discharge. Rubbing dirt on the wire before it was heated seemed to produce no effect on the rate of discharge. The different wires did, indeed, behave differently, and yet if the whole effect had been produced by dirt, the phenomena would not have been at all what they were.

A second explanation is one suggested by Berliner ${ }^{1}$ that the discharge is produced by occluded hydrogen. If we modify this statement and say that the positive discharge is largely produced in this way, much may be said in favor of the suggestion. At lower temperatures the ionization appears to be produced within or very near to the surface of the metal. This part, at least, of the ionization may be produced by the occluded hydrogen.

This explains the fact that the discharge is so much larger when the wire is first heated in a vacuum. The hydrogen which is driven out from the wire would be largely ionized. Heating the wire in hydrogen would, of course, largely restore to it the power to produce a large discharge, and heating it in air would not.

We would expect the gases within the metal to be ionized at the lower temperatures. At higher temperatures the gas surrounding the wire would begin to be ionized. This would produce the ionization which we found could be blown from the wire. This ionization about the wire would allow the negative discharge to occur, since both positive and negative ions would be produced beyond the surface layer in which the fall of potential is supposed to exist.

The ions from the occluded hydrogen would undoubtedly move more rapidly than those produced in the air. In the case of dis- 
charge produced by X-rays the positive ions of the hydogen move more rapidly than the negative of other gases. By supposing that the more rapidly moving positive ions are ions from the occluded hydrogen and that the slower ones are from the air, several other facts could be explained, as, for example, that the average velocity of the ions becomes less at higher temperatures and that the velocity of positive ions in air is greater than the negative, but the action of the particles driven off from the wire would also explain these facts, and it seems more probable that their action is the predominant one.

Possibly the particles which are driven off may ionize the air about the wire and are thus the cause of the slower ions, first, directly, by loading ions already formed, and second, indirectly, by producing a new class of ions.

The fact that a negative discharge in a vacuum is affected by the length of time that the wire is heated shows that the negative discharge is not all produced by the ions about the wire. Apparently some of the discharge is caused by something coming from within the wire.

In all probability neither the positive nor the negative discharge is produced by any one cause. It seems safe to say that the positive ions produced at the lower temperature come from within the wire, and that some at least of the negative ions are produced in the gas about the wire. It is also safe to say that some of the ions that come from within the wire are due to occluded hydrogen, and that particles driven off from the wire produce some of the phenomena. Probably the different causes enter in different amounts with different pieces of wire.

Summary.-The particles producing the decrease in the velocity of the ions come from the hot platinum and not from the copper connections.

The particles from a hot platinum wire have a greater retarding action on the negative ions drawn from a flame than on the positive.

In hydrogen the rate of discharge is much larger than in air. The positive discharge still begins at lower temperatures than the negative. The negative soon surpasses the positive, passes through a maximum and rapidly decreases, coming to have about the same value as the positive when very near the melting point. The 
presence of particles about the wire after it had been heated was also shown by the cloud formation when the hydrogen was suddenly cooled, but no loss in the weight of the wire was detected. Apparently particles are driven off, but to a much smaller extent than in air. The largest value for $K_{1}$ was found to be $42.6 \mathrm{~cm}$. per sec. for a potential gradient of I volt per cm., as compared with $4.5 \mathrm{~cm}$. in air and for $K_{2} 195 \mathrm{~cm}$. as compared with $3.6 \mathrm{~cm}$. As in air the rates of discharge increased very rapidly as the potential differences were increased.

The behavior of the wire in oxygen, $\mathrm{CO}_{2}$ and $\mathrm{CO}$ is practically the same as in air.

The experiments seemed to indicate that the particles are driven off as platinum and not as some oxide of platinum.

When a platinum wire is first heated in a vacuum the rate of discharge is much greater than at any time afterwards. Heating the wire in hydrogen partially restored to it the power of producing a large discharge. But this did not produce any effect on the negative discharge.

The rate of discharge is much greater in a vacuum than in air at atmospheric pressures.

The positive discharge at higher temperatures is less in a vacuum than the negative. The amount of both positive and negative discharges continually increase as the temperature of the wire is increased.

The potential difference between the wire and cylinder about it has little effect on the amount of discharge in a vacuum. In this respect it behaves entirely different from the wire in air at atmospheric pressures. The size of the surrounding cylinder has little effect on the amount of discharge. No method was found by which the velocity of the ions in a vacuum could be determined.

The phenomena indicate that the ionization first occurs within the wire, and there are some reasons for thinking that this part of the ionization is produced from occluded hydrogen. 